\title{
The QWIP focal plane assembly for NASA's Landsat Data Continuity Mission
}

\author{
M. Jhabvala ${ }^{1}$, D. Reuter ${ }^{1}$, K. Choi ${ }^{2}$, M. Sundaram ${ }^{3}$, C. Jhabvala ${ }^{1}$, A. La ${ }^{1}$, \\ A. Waczynski ${ }^{4}$ and J. Bundas ${ }^{3}$ \\ ${ }^{1}$ NASA Goddard Space Flight Center, Greenbelt, Maryland 20771 USA \\ ${ }^{2}$ US Army Research Laboratory, 2800 Powder Mill Road, Adelphi, Maryland, 20783 USA \\ ${ }^{3}$ QmagiQ, LLC, 22 Cotton Road, Unit H, Suite 180, Nashua, NH 03063 USA \\ ${ }^{4}$ Global Science and Technology, 7885 Walker Drive, Greenbelt, MD 20770 USA
}

\begin{abstract}
The Thermal Infrared Sensor (TIRS) is a QWIP based instrument intended to supplement the Operational Land Imager (OLI) for the Landsat Data Continuity Mission (LDCM) [1]. The TIRS instrument is a dual channel far infrared imager with the two bands centered at $10.8 \mu \mathrm{m}$ and $12.0 \mu \mathrm{m}$. The focal plane assembly (FPA) consists of three $640 \times 512 \mathrm{GaAs}$ Quantum Well Infrared Photodetector (QWIP) arrays precisely mounted to a silicon carrier substrate that is mounted on an invar baseplate. The two spectral bands are defined by bandpass filters mounted in close proximity to the detector surfaces. The focal plane operating temperature is 43K. The QWIP arrays are hybridized to Indigo ISC9803 readout integrated circuits (ROICs). Two varieties of QWIP detector arrays are being developed for this project, a corrugated surface structure QWIP and a grating surface structure QWIP. This paper will describe the TIRS system noise equivalent temperature difference sensitivity as it affects the QWIP focal plane performance requirements: spectral response, dark current, conversion efficiency, read noise, temperature stability, pixel uniformity, optical crosstalk and pixel yield. Additional mechanical constraints as well as qualification through Technology Readiness Level 6 (TRL 6) will also be discussed.
\end{abstract}

Keywords: QWIPs, quantum well detectors, infrared focal planes, IR detector arrays, GaAs detectors

\section{INTRODUCTION}

The Landsat Data Continuity Mission is planned to be launched in late 2012 and will continue the 30+ year legacy of the NASA Landsat program. The LDCM is a joint NASA-US Geological Survey (USGS) mission. The Thermal Infrared Sensor is a dual band QWIP-based instrument intended to monitor a variety of earth-based and atmospheric phenomenon: agricultural monitoring; evapotranspiration; cloud detection and analysis; mapping heat fluxes from cities; monitoring air quality; monitoring volcanic activity; monitoring the rain forests; biomass burning; industrial thermal pollution in the atmosphere, rivers and lakes; monitoring/tracking material transport in lakes and coastal regions; identifying insect breeding areas and applications that will ultimately arise in the future as a result of global warming and climate change. Evapotranspiration, is essentially the water evaporation from vegetation and is influenced by water availability, crop species and health and a variety of other agricultural variables [2]. The scarcity of water in the western United States is a serious economic issue and satellite imagery is heavily relied upon to resolve water rights disputes as well as monitoring (legal and illegal) water usage. This problem is only expected to become more of an issue in the future. The NASA Landsat program has been in existence since 1978 and in 1999 Landsat 7 was launched with a design lifetime of 5 years. The Landsat program has provided data on a wide range of earth science parameters and is an international resource. The Landsat Data Continuity Mission is intended to keep this resource available with a launch date scheduled for 2012. In order to provide a more comprehensive data set on a variety earth science parameters it was determined that thermal imagery in the 10.5-12.5 $\mu \mathrm{m}$ spectral range was essential. The Operational Land Imager (OLI), also aboard LDCM, will provide spectral coverage in the visible and near infrared ( $433 \mathrm{~nm}$ to $1.39 \mu \mathrm{m})$ in discrete bands. 
The decision to include a thermal imager was not finalized until late 2008 and so many elements had to be considered in the selection of the focal plane detector such as delivery schedule, array and readout format options, operating temperature, technology maturation and technological reliability, cost, to list a few of the key considerations. In the past, QWIP based focal planes have not been considered either because of a lack of technological heritage or inadequate performance for the specific mission. The low conversion efficiency of QWIP devices combined with the proven heritage of $\mathrm{HgCdTe}$ and InSb technologies have routinely disqualified QWIPs from being considered. However, the requirements of the TIRS instrument presented a unique opportunity for implementing a QWIP focal plane: compatibility with the TIRS science specifications, previously developed and tested array format, readout integrated circuit (ROIC) availability, multiple QWIP array fabrication sources, a focal plane design with proven reliability [3] and cost/schedule mission compatibility. The relative simplicity (in a high technology world) of QWIP design and fabrication has been proven and documented [4]. This advantage of QWIP technology has been largely responsible for the rapid advancement from single element detectors 20 years ago [5] to arrays exceeding 1 million pixels [6]. The compatibility with silicon based processing facilities has led to the proliferation of research and development in laboratories throughout the world, from university labs to large aerospace companies. However, in order to be considered in a NASA mission a much more stringent technology readiness level (TRL) must be demonstrated. These instruments tend to be unique, very expensive, generally only have one chance to succeed and must be reliable for long periods of time in a hostile space environment. These instruments must survive the rigors of a launch including severe vibration, radiation effects and often a shock event (such as the explosive removal of a protective cover). There is no room for chance or even the slightest error and this is after the fundamental technologies have been thoroughly proven. This paper will describe the TIRS system noise equivalent temperature difference sensitivity as it affects the QWIP focal plane performance requirements: spectral response, dark current, conversion efficiency, read noise, temperature stability, pixel uniformity, optical crosstalk and pixel yield. Mechanical constraints and qualification through Technology Readiness Level 6 (TRL 6) will also be discussed.

\section{DERIVED FOCAL PLANE REQUIREMENTS}

The LDCM satellite will be launched into in a sun synchronous polar orbit at an altitude of $705 \mathrm{~km}$ with an instantaneous field of view (IFOV) of $100 \mathrm{~m} \times 100 \mathrm{~m}$ per pixel and a total ground sample distance of $185 \mathrm{~km}$ for the focal plane. This corresponds to a pixel line width of 1850 pixels in the horizontal direction. To meet this requirement three individual detector arrays, each $640 \times 512$ pixels, was determined to best satisfy the mission requirements while relying on existing and manageable technologies (as opposed to designing a customized array and ROIC). The Indigo ISC9803 [7] was selected as the ROIC. The arrays are co-registered on a common substrate and the imager is operated in a push-broom fashion. The mission and science requirements determine the necessary QWIP array performance requirements as shown in Table 1.

The TIRS system model was developed to include the equivalent noise inputs from all potential sources on the instrument. Most importantly are the effects from elevated component temperatures and the temperature instabilities associated with these components. The total noise equivalent model is expressed as:

$$
\begin{aligned}
& I_{\text {all sources }}^{2}=i^{2} \text { shot, Id }+i^{2} \text { shot, signal }+i_{\Delta T, \text { Id }}^{2}+i_{\Delta T \text {, tel. bkgrnd }}{ }^{2} i_{\Delta T}^{2} \text {, mirror }+i^{2}{ }_{\Delta T} \text {, tel. optics } \\
& +i_{\text {ROIC read }}^{2}+i_{\text {elec, noise }}+i^{2} \text { A/D noise }
\end{aligned}
$$

where,

$$
\begin{aligned}
& \mathbf{i}^{2} \text { shot, Id } \\
& \mathbf{i}^{2} \text { shot, signal } \\
& \mathbf{i}^{2} \Delta T, \text { Id } \\
& \mathbf{i}^{2} \Delta T \text {, tel. bkgrnd } \\
& \mathbf{i}^{2} \Delta T \text {, mirror } \\
& \mathbf{i}^{2} \Delta T \text {, tel. optics } \\
& \mathbf{i}^{2} \text { ROIC read } \\
& \mathbf{i}^{2} \text { elec, noise } \\
& \mathbf{i}^{2} \text { A/D noise }
\end{aligned}
$$

is the dark current shot noise is the shot noise from the target $(300 \mathrm{~K})$ is the noise resulting from the dark current variation due to temperature instability is the noise resulting from the telescope temperature instability is the noise resulting from the telescope mirror instability is the noise resulting from the telescope optics instability is the ROIC read noise is the added focal plane electronics noise is the $\mathrm{A} / \mathrm{D}$ quantization noise 
The noise equivalent irradiance, $\mathrm{NE} \Delta \mathrm{I}$, based on the Landsat mission requirements is:

$$
\begin{array}{ll}
\text { Band } 1 & <0.059 \mathrm{~W} / \mathrm{m}^{2}-\mathrm{sr}-\mu \mathrm{m} \\
\text { Band } 2 & <0.049 \mathrm{~W} / \mathrm{m}^{2}-\mathrm{sr}-\mu \mathrm{m}
\end{array}
$$

Table 1. System Requirements

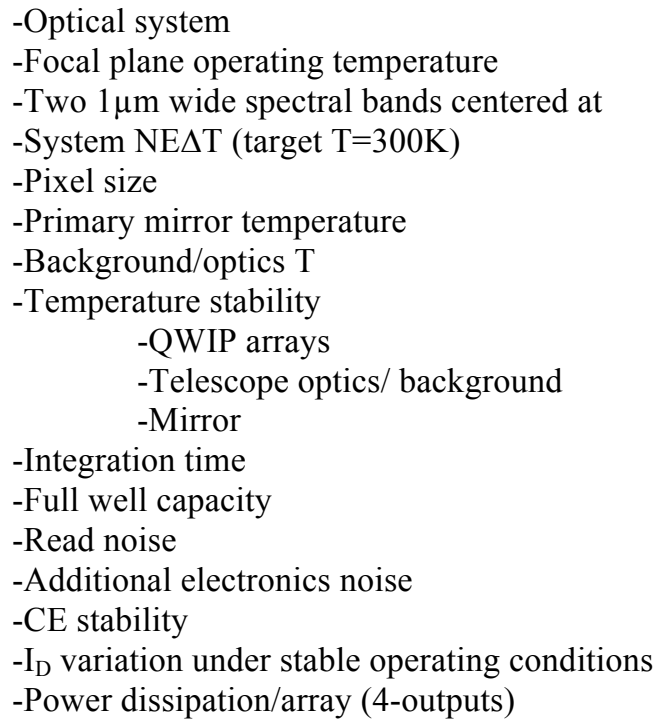

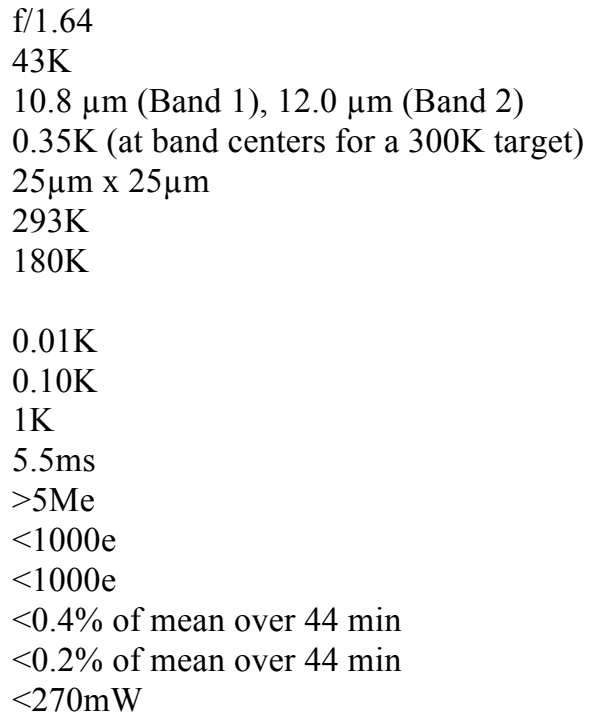

The major contributions to the elevated NE $\Delta \mathrm{T}$ arise from the focal plane temperature instability as it affects the dark current stability and similarly the telescope background temperature instability. Consequently, the temperature stability of these two regions as been severely constrained. The ground sample distance requirement of $185 \mathrm{~km}$ with an IFOV of $100 \mathrm{~m}$ requires 1,850 pixels per swath width (across track, or $\mathrm{x}$, direction). To meet this requirement three $640 \mathrm{x} 512$ arrays are needed totaling 1,920 pixels in the $\mathrm{x}$, or horizontal, focal plane direction. This allows for 70 columns to be utilized for array overlap and edge column exclusion. One further key specification resulting from the push-broom operation of the imager is:

Within each filter band on each Sensor Chip Array (SCA), the FPA shall provide at least 3 unique pixel rows which can be combined through ground processing so that any combination of 2 rows has fewer that $0.1 \%$ of the pixel elements that fail to meet operability requirements for any continuous data collection period up to 44 minutes.

The $0.1 \%$ part of this specification translates to essentially one pixel out of the 1,850 that is allowed to be out of specification. Our goal, and one of the advantages of QWIP technology in general, is to meet this specification by obtaining rows with $100 \%$ full specification compliance (that is, $0 \%$ versus $0.1 \%$ ). This specification also dictates that we need to yield only a small number of rows from the 512 rows available per array.

The NE $\Delta \mathrm{I}$ requirements determine the system or instrument NE $\Delta \mathrm{T}$ requirements (not to be confused with the detector $\mathrm{NE} \Delta \mathrm{T}$ ). The corresponding system NE $\Delta \mathrm{T}$ requirements are $0.35 \mathrm{~K}$ viewing a $320 \mathrm{~K}$ target and $0.40 \mathrm{~K}$ viewing a $300 \mathrm{~K}$ target at both $\lambda=10.8 \mu \mathrm{m}$ and $12.0 \mu \mathrm{m}$. However, in addition to all other system level margins we have further tightened this specification in our QWIP performance models to:

System NE $\Delta \mathrm{T} \leq 0.33 \mathrm{~K}$ at all wavelengths from $10.5-12.3 \mu \mathrm{m}$ (for a $300 \mathrm{~K}$ source).

The number of photons emitted from a blackbody source per unit time per unit solid angle and arriving at a detector of unit area is given by: 


$$
\mathrm{Q}(\lambda)=\mathrm{B} / \pi\left[\int_{\lambda_{1}}^{\lambda_{2}} \lambda^{-4} \cdot\left(e^{\mathrm{c} 2} / \lambda \cdot \mathrm{T}_{\mathrm{i}}-1\right)^{-1} \mathrm{~d} \lambda\right]
$$

where,

$\mathrm{T}$ is the source or target temperature $(\mathrm{K})$

$\lambda$ is the spectral wavelength

$\mathrm{B}=1.88 \cdot 10^{23} \mathrm{~m}^{3} / \mathrm{cm}^{2}-\mathrm{sec}$

$\mathrm{c}_{2}=1.44 \cdot 10^{4} \mathrm{~m}-\mathrm{K}$

The number of signal electrons generated by a pixel is then;

$$
\mathrm{S}_{\mathrm{Ti}}=\Omega \cdot \mathrm{t}_{\text {int }} \cdot \mathrm{T}_{\mathrm{opt}} \cdot \mathrm{A}_{\mathrm{D}} \cdot \mathrm{g} \cdot \eta \cdot \mathrm{Q}(\lambda)=\Omega \cdot \mathrm{t}_{\text {int }} \cdot \mathrm{T}_{\mathrm{opt}} \cdot \mathrm{A}_{\mathrm{D}} \cdot \mathrm{g} \cdot \eta \cdot \frac{\mathrm{B}}{\pi}\left[\int_{\lambda_{1}}^{\lambda_{2}} \lambda^{-4} \cdot\left(e^{\mathrm{c}_{2} / \lambda \cdot \mathrm{T}_{\mathrm{i}}}-1\right)^{-1} \mathrm{~d} \lambda\right]
$$

where,

$S_{T i}$ the \# of electrons generated by the pixel from a source at temperature $T_{\mathrm{i}}$ in the spectral band $\lambda_{1}-\lambda_{2}$

$\Omega$ is the solid angle viewed by the pixel

$t_{\text {int }}$ is the signal integration time

$\mathrm{T}_{\text {opt }}$ is the complete optical transmission of the system

$A_{D}$ is the pixel area

$\mathrm{g}$ is the photoconductive gain

$\eta$ is the absorptive quantum efficiency of the detector

The solid angle, $\Omega$ is defined by the telescope $\mathrm{f} / \#$ and is

$$
\Omega=\pi /\left(1+4 \mathrm{f}^{2}\right)
$$

The figure of merit typically used to describe the photon to electron conversion efficiency, CE, is

$$
\mathrm{CE}=\mathrm{g} \cdot \eta
$$

The system NE $\Delta \mathrm{T}$ can now be calculated based on the TIRS system elements and stability from:

$$
\mathrm{NE} \Delta \mathrm{T}=\frac{\left[\Sigma \mathrm{I}_{\mathrm{n}}^{2}\right]^{1 / 2}}{\mathrm{~S}_{\Delta \mathrm{T}}}
$$

$\mathrm{S}_{\Delta \mathrm{T}}$ is calculated by varying the temperature in equation (3) by $1 \mathrm{~K}$. For the nominal $12.0 \mu \mathrm{m}$ band, the TIRS operating conditions are;

$$
\lambda_{1}=11.5 \mu \mathrm{m} ; \lambda_{2}=12.5 \mu \mathrm{m}: \mathrm{T}_{1}=300 \mathrm{~K} ; \mathrm{T}_{2}=301 \mathrm{~K}(\Delta \mathrm{T}=1 \mathrm{~K}) ; \mathrm{T}_{\text {opt }} \approx 0.49^{`} ; \mathrm{g}=0.3 ; \mathrm{t}_{\mathrm{int}}=5.5 \mathrm{~ms} ; \mathrm{A}_{\mathrm{D}}=6.25 \mathrm{E}-4 \mathrm{~cm}^{2}
$$

Based on the system parameters the noise contribution from the various sources can be calculated. The contribution from the various sources based on these nominal TIRS operating conditions is shown below in table 2. It is apparent that for this instrument some effects dominate the system performance. Measured CE and dark current, set by adjusting the detector bias, for the calculated values in Table 2 are;

$$
\begin{aligned}
& \mathrm{CE}=0.8 \% \\
& \mathrm{I}_{\mathrm{D}}=4 \times 10^{7} \mathrm{e} / \mathrm{s}
\end{aligned}
$$

The number of signal electrons for a $1 \mathrm{~K}$ change in temperature for a $300 \mathrm{~K}$ source is:

$$
\mathrm{S}_{\Delta \mathrm{T}}=2.52 \times 10^{4} \mathrm{e}
$$


Table 2. TIRS noise parameters

\begin{tabular}{|c|c|}
\hline Noise source & TIRS nominal value $\left(\mathrm{e}^{2}\right)$ \\
\hline $\mathbf{i}^{2}$ shot, Id & $1.32 \times 10^{5}$ \\
\hline $\mathbf{i}^{2}$ shot, signal & $1.67 \times 10^{6}$ \\
\hline $\mathbf{i}^{2} \Delta \mathrm{T}, \mathrm{Id}$ & $1.25 \times 10^{6}$ \\
\hline $\mathbf{i}^{2} \Delta \mathrm{T}$, tel. bkgrnd & $9.41 \times 10^{6}$ \\
\hline $\mathbf{i}^{2} \Delta \mathrm{T}$, mirror & $1.58 \times 10^{6}$ \\
\hline $\mathbf{i}^{2} \Delta \mathrm{T}$, tel. optics & $1.14 \times 10^{4}$ \\
\hline $\mathbf{i}^{2}$ RoIC read & $3.03 \times 10^{5}$ \\
\hline $\mathbf{i}^{2}$ elec, noise & $10^{6}$ (specified) \\
\hline $\mathbf{i}^{2} \mathrm{~A} / \mathrm{D}$ noise & $4.87 \times 10^{5}$ \\
\hline$\left[\Sigma \mathbf{I}^{2}\right]^{1 / 2}$ & $3.98 \times 10^{3}$ \\
\hline
\end{tabular}

The system NE $\Delta \mathrm{T}$ is calculated as:

$$
\mathrm{NE} \Delta \mathrm{T}=\frac{\left[\Sigma \mathrm{I}_{\mathrm{n}}^{2}\right]^{1 / 2}}{\mathrm{~S}_{\Delta \mathrm{T}}}=\frac{3.98 \times 10^{3}}{2.52 \times 10^{4}}=0.16 \mathrm{~K}
$$

The QWIP array NE $\Delta \mathrm{T}$ can be determined by setting the noise terms unrelated to the detector/ROIC to zero. This will be further discussed.

\section{FOCAL PLANE ARCHITECTURE}

Shown below in figure 1 is the TIRS instrument assembly and the Observational Land Imager (OLI) on which the TIRS instrument is attached.
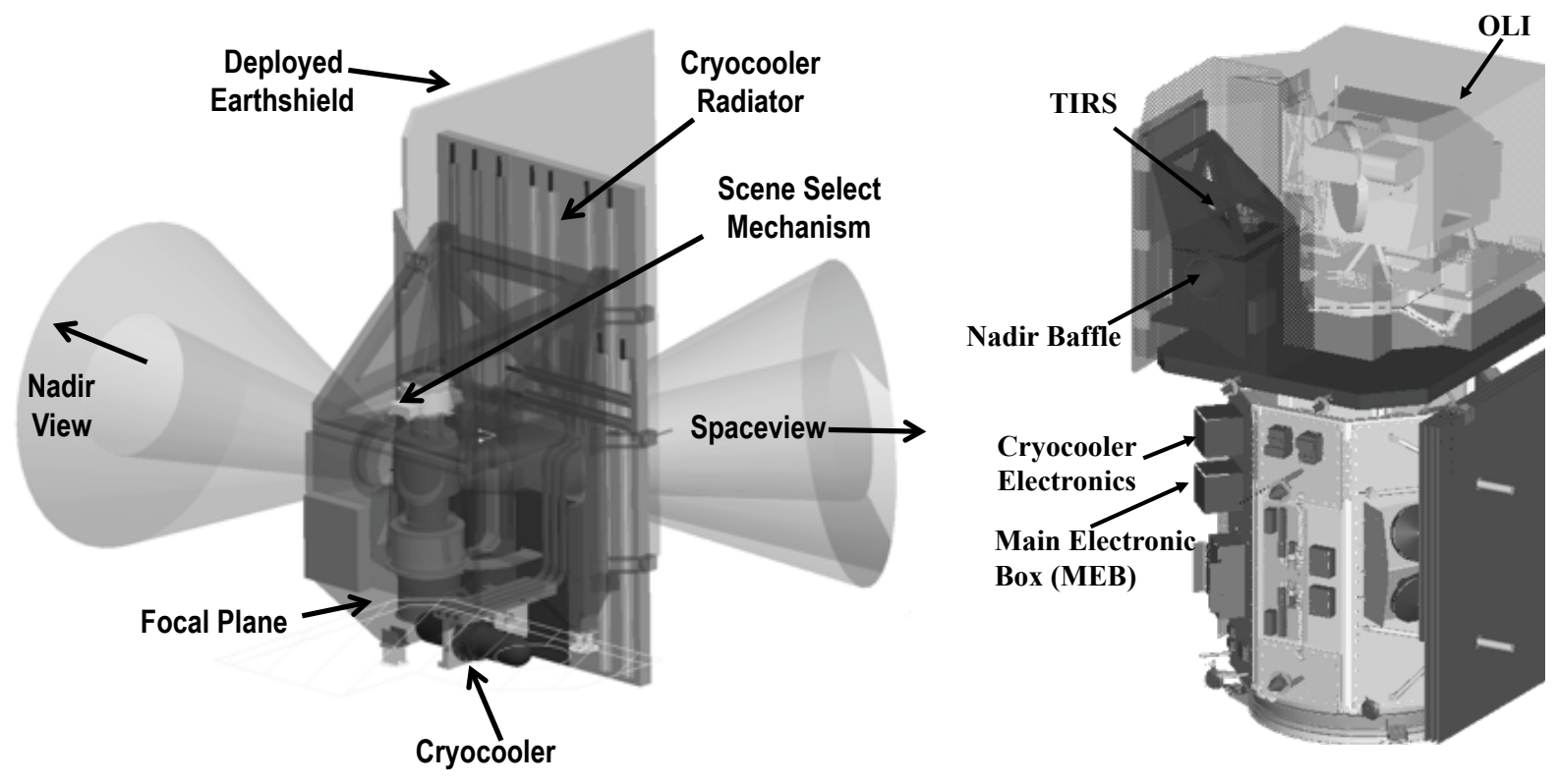

Figure 1. The TIRS instrument assembly (left) and the TIRS instrument accompanying the OLI. 
The OLI is a $0.433-1.39 \mu \mathrm{m}$ imager intended to continue the Landsat spectral coverage of the earth. TIRS is the thermal imaging supplement to this mission. TIRS is designed to operate as a push-broom scanner where a 100 meter swath width of the image is captured in a single row of pixels on the focal plane. As the satellite orbits, the row data is sequentially collected in synchronization with the progression of the satellite over the earth. The ground scene is then reconstructed (on the ground) as a full image. Each row of data corresponds to a strip of earth scenery $100 \mathrm{~m} \mathrm{x} 1850 \mathrm{~m}$. There are two filter bands so the transmitted data set consists of a row corresponding to a $1 \mu \mathrm{m}$ spectral bandwidth centered at $10.8 \mu \mathrm{m}$ and another row of data $1 \mu \mathrm{m}$ in bandwidth centered at $12.0 \mu \mathrm{m}$. Additionally, rows of dark (blanked off) pixels are also collected for dark current calibration and analysis. The focal plane design and layout is shown in figure 2.
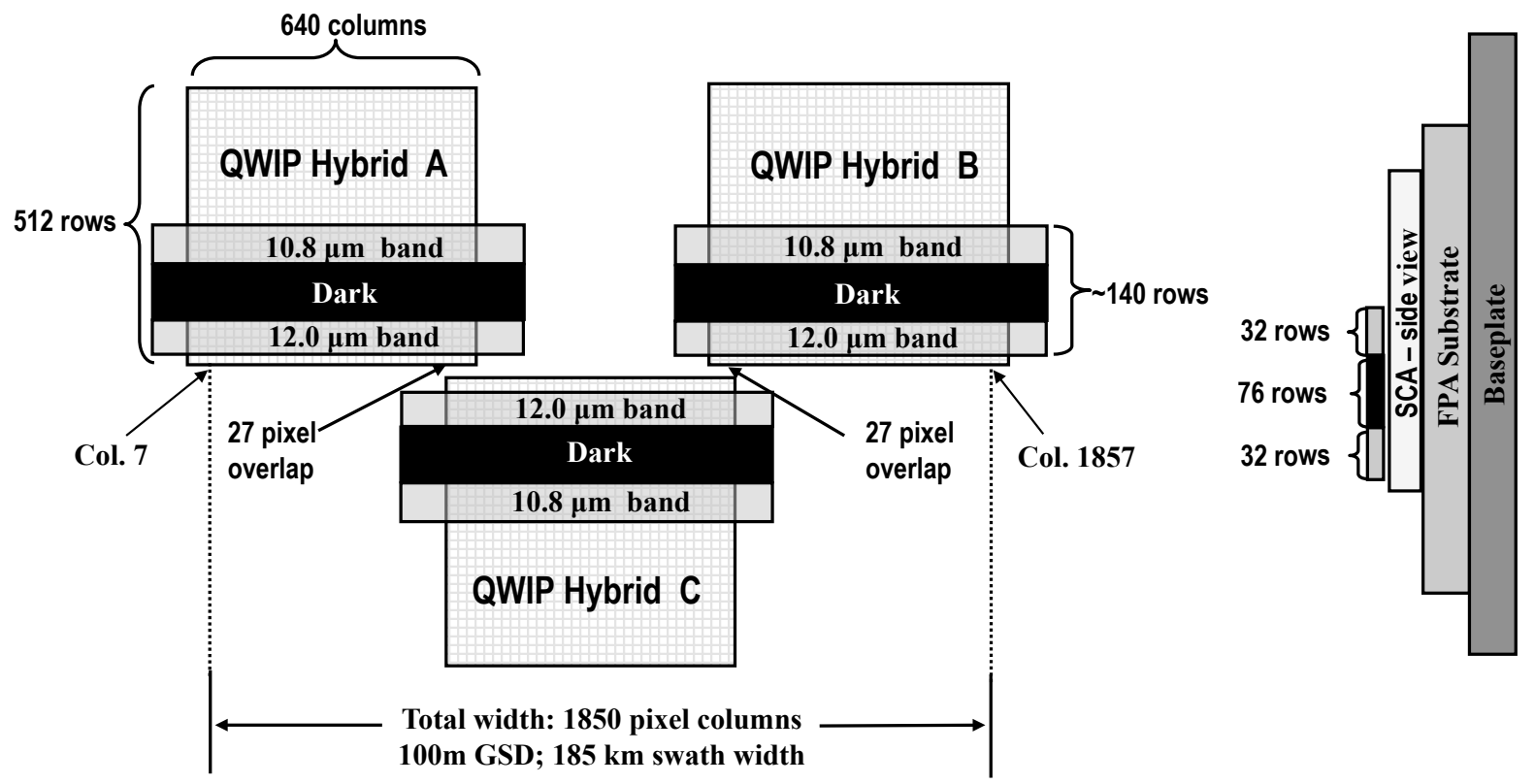

Figure 2. The TIRS focal plane design showing the position of the three QWIP hybrids and the filter bands (left) and a cross sectional view of the focal plane construction (right).

The three arrays are precisely aligned to each other in the horizontal and vertical directions (to within $2 \mu \mathrm{m}$ ). There is a requirement that the detection region within the QWIP array be within $10 \mu \mathrm{m}$ of a common focal plane altitude. This specification is challenging since it includes surface non-uniformities of the baseplate, substrate, the QWIP/ROIC hybrid and the epoxy bond lines between these components. Nonetheless, since there are three discreet arrays they must all fall within a single focus position. The filter bands are further confined to specific regions of the QWIP array. Although each array contains 512 rows, after all the operational requirements are satisfied (frame rate, windowing, co-registration, scene reconstruction, etc.) only 32 rows are available under each filter band separated by 76 rows of occluded pixels (for dark current subtraction). Once all these requirements are incorporated into the focal plane design, eligible rows on any given array are pre-determined. Of these eligible rows, there must be three that can be combined to make two perfect rows, or preferably, at least two perfect rows (that is, rows where all pixels meet every specification). Figure 3 illustrates the specific row "harvesting" options available on any given array. Only the top 160 rows can be considered when screening the arrays. Figure 4 shows the both the physical and electrical layout of the hybrids on the common substrate. Figure 5 is a photograph of the focal plane with the filter assembly installed and aligned over the QWIP hybrids. Two approaches to developing the QWIP arrays are being pursued. One is a design and fabrication based on the corrugated or C-QWIP [8], the other is a design and fabrication based on a surface grating [9]. Each approach has its advantages but the decision as to which arrays best satisfy the mission requirements takes into consideration all interacting aspects of the instrument such as; the over all system NE $\Delta \mathrm{T}$ performance, temperature stability of the 


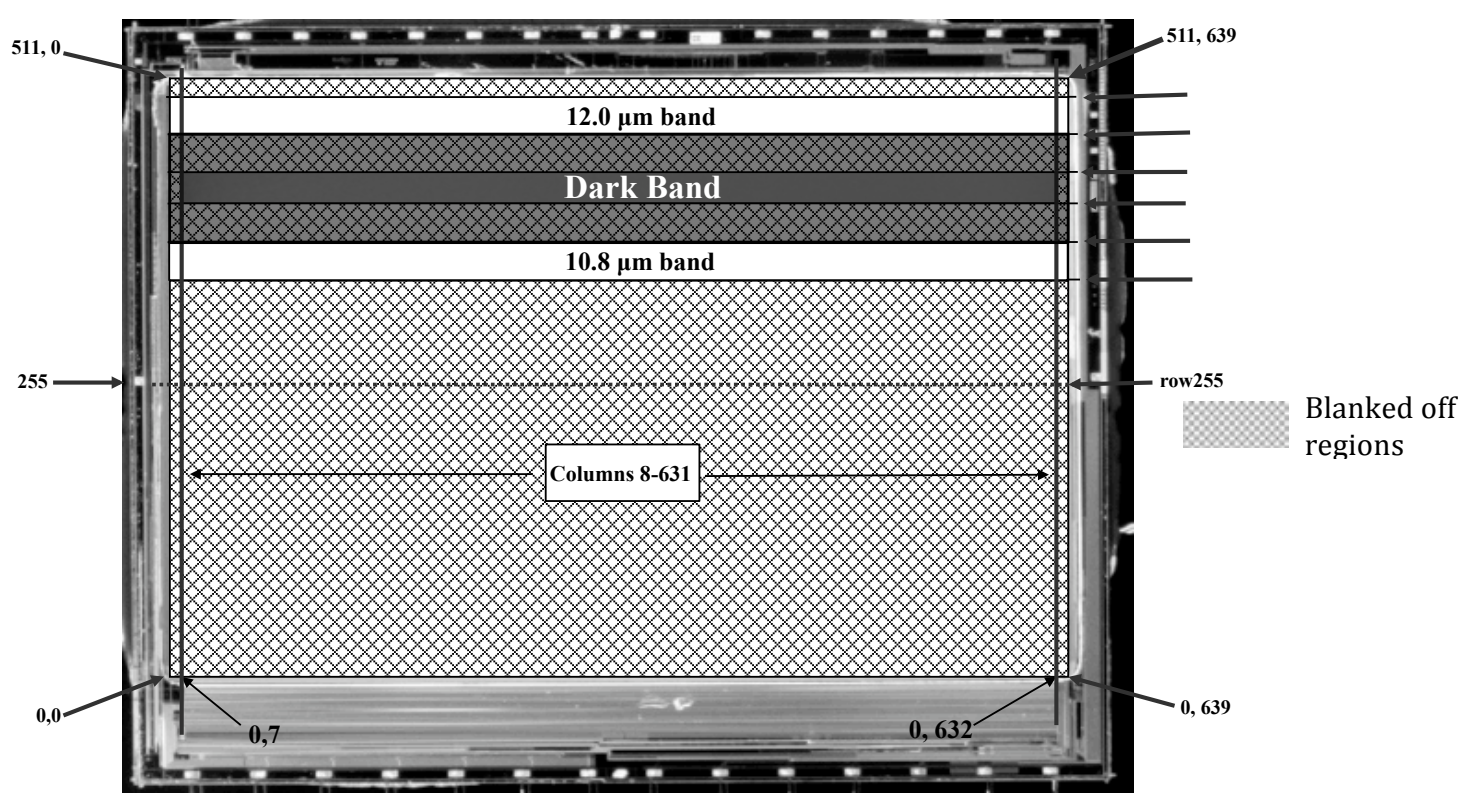

Figure 3. Identification of the specific rows on the QWIP hybrid available for science utilization in the TIRS instrument.
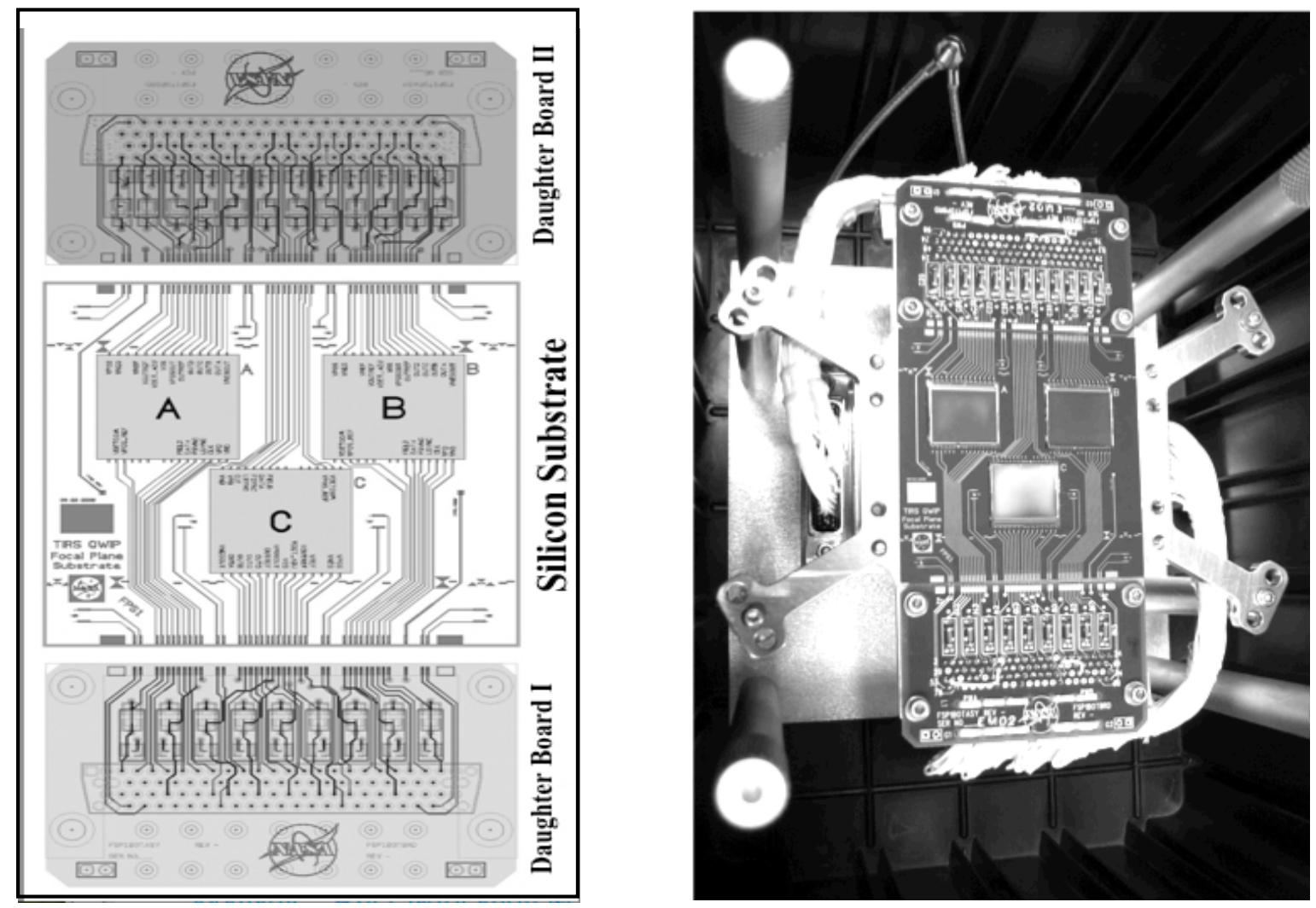

Figure 4. Left; Electrical layout of the three QWIP hybrids and the electrical interface. Right; Photograph of the focal plane without the overlaying filter assembly. 


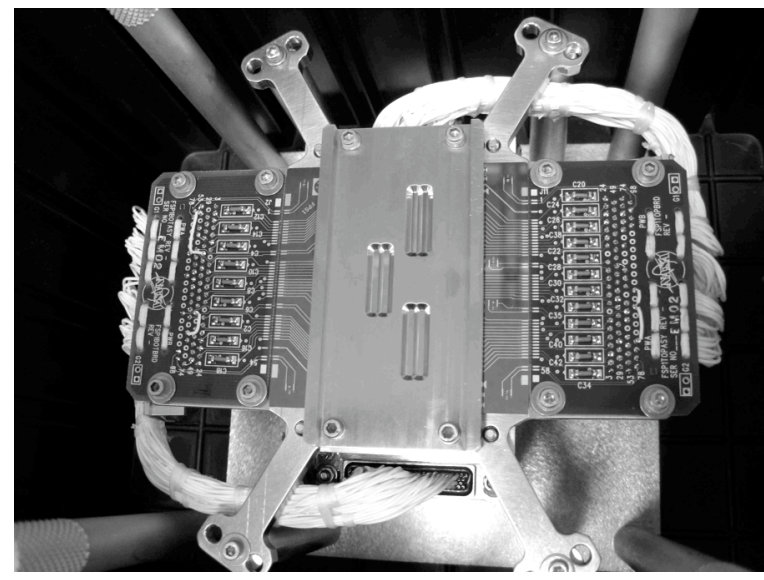

Figure 5. Focal plane assembly with filter assembly installed and aligned.

instrument (which determines the dark current requirement), spectral response (which impacts the filter element design), spectral uniformity across the array and the conversion efficiency stability and dark current stability over 44 minutes. The grating-based QWIP and the C-QWIP were fabricated on GaAs wafers where the respective epitaxial growth recipes were different primarily in the number of quantum wells in the superlattice stack. To achieve the long wavelength response the aluminum mole fraction was reduced to $<17 \%$. Many arrays were fabricated of each variety and carefully screened. The final selection of the flight arrays is yet to be determined although there is adequate numbers of each type that meet the system NE $\Delta T$ requirement. There are noteworthy differences between the two types of QWIP arrays and their respective characteristics have been extensively described in the literature. Scanning electron microscope images of the surface of each type of QWIP array are shown in figure 6. QmagiQ, LLC, fabricated the grating QWIP array and the C-QWIP was jointly fabricated by ARL and NASA/GSFC. As the development progressed the three groups further improved the fabrication process by best utilizing each groups skills and resources (a collaboration that was, and continues to be, essential for a successful outcome).
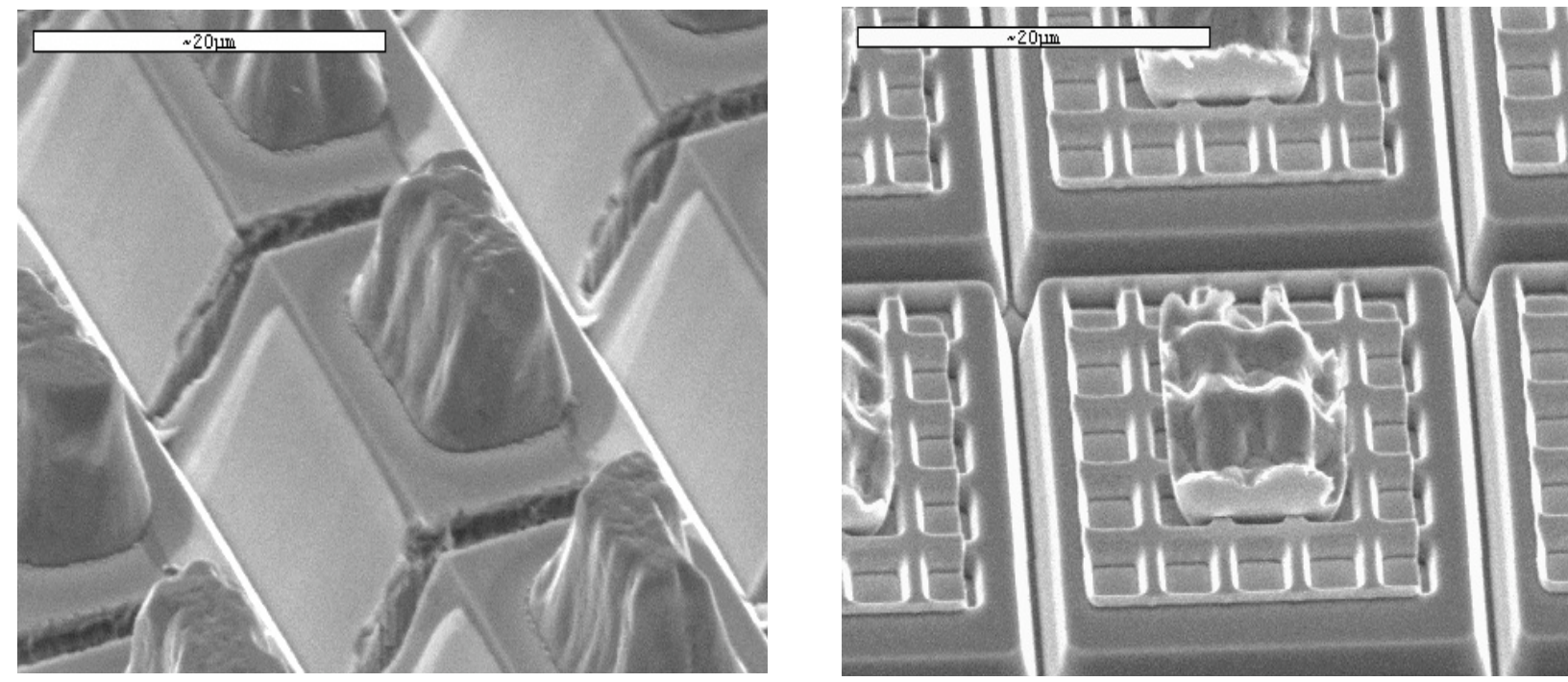

Figure 6. Scanning electron microscope images of the C-QWIP (left) and grating-based QWIP surface (right).

\section{TEST RESULTS}

Extensive tests were performed on many QWIP hybrids including; quick-look imaging test, relative spectral response, absolute CE, dark current, read noise, spectral uniformity, dark current stability and CE stability over 44 minutes, row selection for each band, array pixel map, full well determination and power dissipation. Additionally, optical crosstalk experiments were performed using a custom fabricated a pinhole mask on a QWIP hybrid, thermal cycling a complete focal plane assembly (consisting of 3 QWIP hybrids) from $300 \mathrm{~K}$ to $77 \mathrm{~K}$ and back to $300 \mathrm{~K}$ for 40 cycles, thermal model verification and subjecting a focal plane assembly to qualification levels of vibration to simulate the Atlas 5 launch $(+3 \mathrm{db})$. The location of these tests involves multiple labs at Goddard including the Detector Characterization Laboratory for the comprehensive performance testing, the QWIP camera laboratory, the optics, cryogenic, and environmental tests facilities as well as labs at both QmagiQ and the Army Research Laboratory. 


\subsection{Quick-look imaging test}

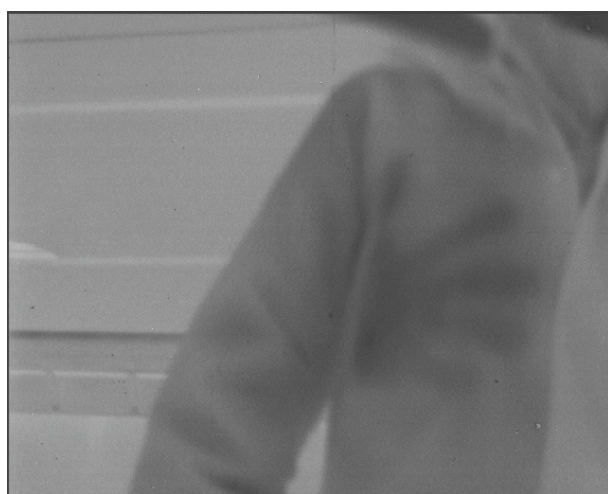

Figure 7. Image demonstrating functionality of the QWIP array.
An initial imaging test is performed with each array in a cryocooled camera system developed for hyperspectral imaging. The array is temporarily mounted in a leadless chip carrier and can be installed, cooled, tested and warmed back to $300 \mathrm{~K}$ in less than a day. This is an essential gate each array must pass prior to the detailed parametric tests performed in the Detector Characterization Laboratory (DCL) at Goddard. An example of such an image is shown in figure 7. The images provide a subjective basis for determining if the array is worthy of further comprehensive testing. Once this determination is made the subsequent tests are far more detailed and time consuming. The DCL has multiple dewar systems for performing the radiometric and static tests such as establishing operating parameters, dark current, power dissipation and read noise measurements, for example. Calibrated, National Institutes of Standards and Tests (NIST) traceable detectors are utilized for the absolute measurements.

\subsection{Conversion efficiency}

The conversion efficiency as a function of the spectral wavelength was measured as a function of the detector bias at $43 \mathrm{~K}$. The measurement was made with a calibrated monochromator in $0.2 \mu \mathrm{m}$ steps from 6 to $15 \mu \mathrm{m}$. Shown in figure 8 below are two spectra from the most recent wafer lots of QmagiQ and GSFC/ARL. These devices were fabricated from entirely different $\mathrm{GaAs}$ wafers with different superlattice structures.
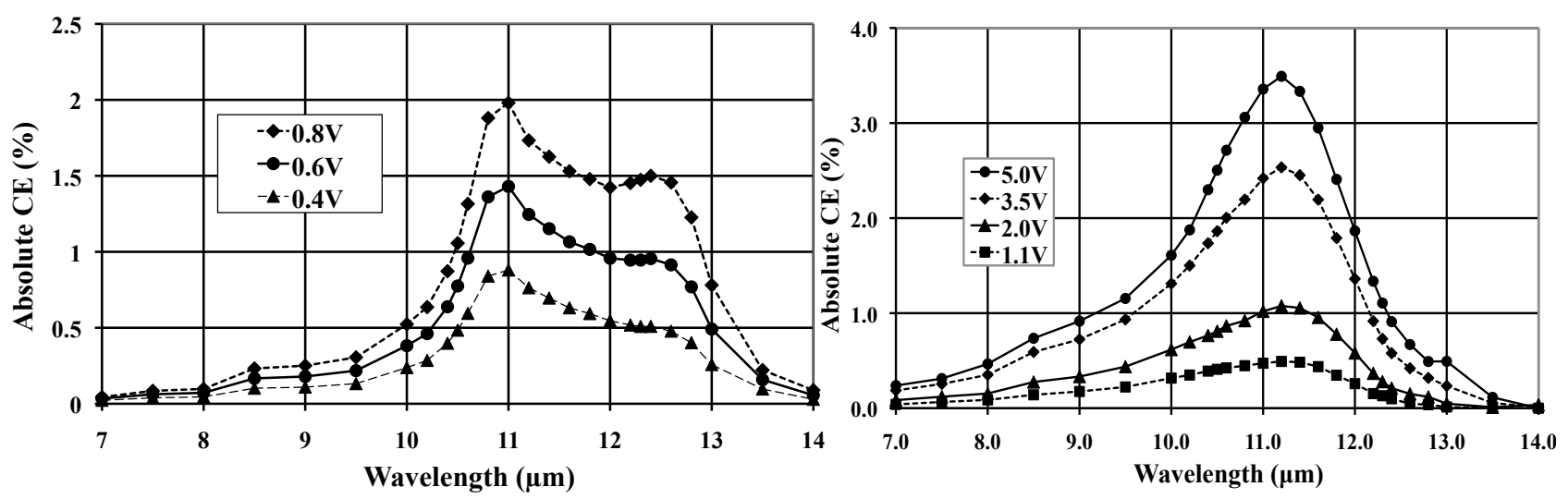

Figure 8. The absolute conversion efficiency as a function of wavelength and QWIP bias voltage for a grating-based (left) and corrugated (right) QWIP detectors. The C-QWIP has more quantum wells and so requires larger bias voltage.

The dependency of the conversion efficiency on the applied detector bias is very apparent from both of these plots. However, with increased bias also comes increased dark current and so there is an optimum operating point which is highly dependant on the overall system parameters. Figure 9 illustrates the effect of both bias voltage and temperature on the dark current for each of the same QWIP arrays shown in figure 8. If the dark current is not a concern then the conversion efficiency can be maximized by increasing the bias to the maximum of either the QWIP pixel (in the case of the QmagiQ grating QWIP) or to the maximum provided by the ROIC (as in the case of the C- QWIP). In this case one can calculate the NE $\Delta \mathrm{T}$ around the peak wavelength, using the average $\mathrm{CE}$ of the response around the peak $( \pm 0.5 \mu \mathrm{m})$ and disregarding the noise effects from the other instrument subsystems. Under these conditions the NE $\Delta \mathrm{T}$ for the QWIP is based on the same signal but only the signal and dark current shot noise and the ROIC read noise is considered. In the case of the TIRS instrument the current minimum operating temperature is expected to be $43 \mathrm{~K}$ (but depending on the cryocooler performance, a lower temperature may be achieved). Based on the above data the QWIP-specific "best- 
case" NE $\Delta \mathrm{T}$ can be calculated based on the assumptions identified in Table 3. The read noise for the ISC9803 is consistently around 260 electrons and is independent of the applied detector bias.
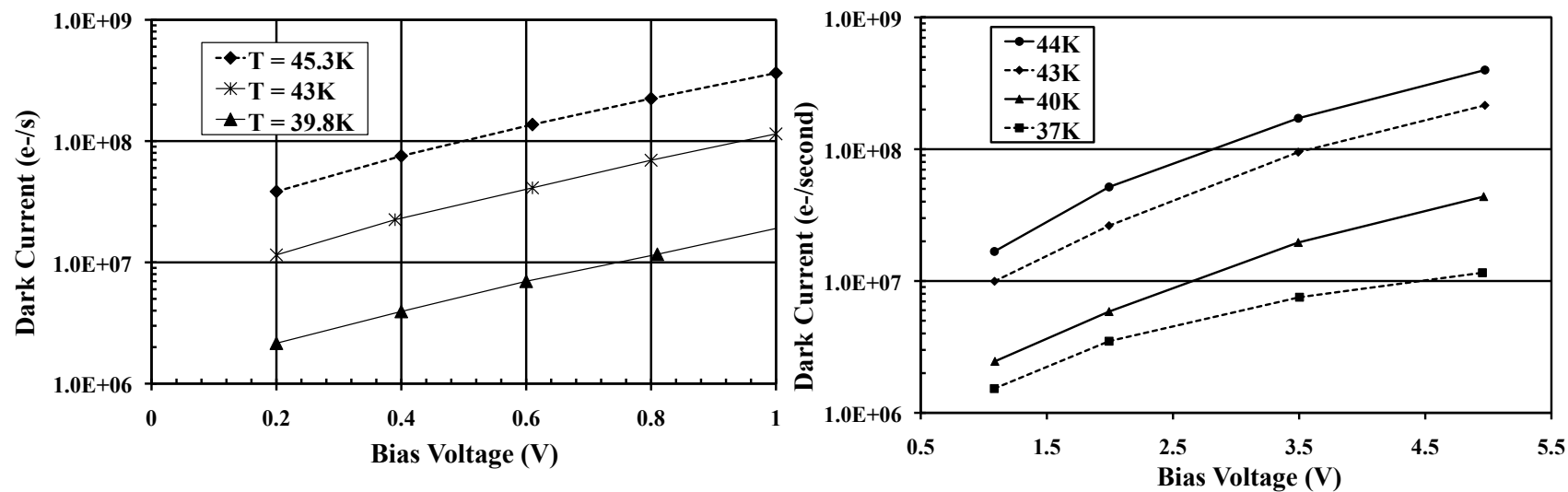

Figure 9. Dark current as a function of bias at selected temperatures. Grating-based QWIP on the left, C-QWIP on the right. In both cases these are the same device as in figure 8 .

Table 3. QWIP array NEAT determination

\begin{tabular}{|l|l|l|}
\hline Parameter & Grating QWIP & C-QWIP \\
\hline Temperature & $43 \mathrm{~K}$ & $43 \mathrm{~K}$ \\
\hline Detector bias & $0.8 \mathrm{v}$ & $3.5 \mathrm{v}$ \\
\hline Photoconductive gain, $\mathrm{g}$ & 0.3 & 0.3 \\
\hline Dark current, $\mathrm{I}_{\mathrm{d}}$ & $8 \mathrm{E} 7 \mathrm{e} / \mathrm{s}$ & $1 \mathrm{E} 8 \mathrm{e} / \mathrm{s}$ \\
\hline Integration time, $\mathrm{t}$ & $5.5 \mathrm{~ms}$ & $5.5 \mathrm{~ms}$ \\
\hline Peak $\lambda$ & $11.0 \mu \mathrm{m}$ & $11.3 \mu \mathrm{m}$ \\
\hline Average CE near peak $\lambda(10.5-11.5 \mu \mathrm{m})$ & .017 & 0.022 \\
\hline Measured ROIC read noise, $\mathrm{I}_{\mathrm{r}}$ & $260 \mathrm{e}-$ & $260 \mathrm{e}-$ \\
\hline $301 \mathrm{~K}$ signal from source, $\mathrm{I}_{\mathrm{s}}(10.5-11.5 \mu \mathrm{m})$ & $4.916 \mathrm{E} 8 \mathrm{ph}$ & $4.916 \mathrm{E} 8 \mathrm{ph}$ \\
\hline $300 \mathrm{~K}$ signal from source, $\mathrm{I}_{\mathrm{s}}(10.5-11.5 \mu \mathrm{m})$ & $4.844 \mathrm{E} 8 \mathrm{ph}$ & $4.844 \mathrm{E} 8 \mathrm{ph}$ \\
\hline $\begin{array}{l}\text { QWIP output signal for } 1 \mathrm{~K} \text { change in source } \\
\text { T }(\mathrm{TIRS} \text { optical system, } 300 \mathrm{~K} \text { source; } 10.5-\end{array}$ & $1.22 \mathrm{E} 5 \mathrm{e}-$ & $1.58 \mathrm{E} 5 \mathrm{e}-$ \\
$11.5 \mu \mathrm{m}), \mathrm{S}$ & & \\
\hline $\begin{array}{l}\text { Total noise contribution, } \mathbf{N}: \\
\left.\left[2 \mathrm{~g}\left(\mathrm{I}_{\mathrm{d}}+\mathrm{CE} \cdot \mathrm{I}_{\mathrm{s}}\right)\right] \mathrm{t}+\mathrm{I}_{\mathrm{r}}^{2}\right]^{1 / 2}\end{array}$ & $2.28 \mathrm{E} 3 \mathrm{e}-$ & $2.60 \mathrm{E} 3 \mathrm{e}-$ \\
\hline Average NE$\Delta \mathrm{T}$ at $\lambda$ peak, N/S & $19 \mathrm{mK}$ & $17 \mathrm{mK}$ \\
\hline
\end{tabular}

It is clear from the calculated $\mathrm{NE} \Delta \mathrm{T}$ values that the arrays are not the limiting performance element in the TIRS instrument. However, in order to mitigate system level effects from other noise sources less than optimum detector operation may actually be beneficial. One example may be to reduce the dark current and hence the CE by applying a lower than optimum detector bias thereby reducing the noise affect from focal plane temperature instability. Trade-offs 
such as these can only be made once the entire instrument is assembled, configured and tested. Nonetheless, this noise margin provided by the QWIP arrays is very valuable in overall performance risk mitigation.

\subsection{Array row selection}

The goal of the array screening process is to identify rows where every pixel in that row meets the specifications and are within the bands defined in figure 3. Shown below in figure 10 are plots for specific rows where $100 \%$ of the pixels meet the system NE $\Delta \mathrm{T}$ requirements. Also included in this selection criteria are: full well exceeds 5 million electrons, array power dissipation $<270 \mathrm{~mW}$ and is typically $\sim 150 \mathrm{~mW}$, read noise $<1000$ electrons and is typically $\sim 260$ electrons.
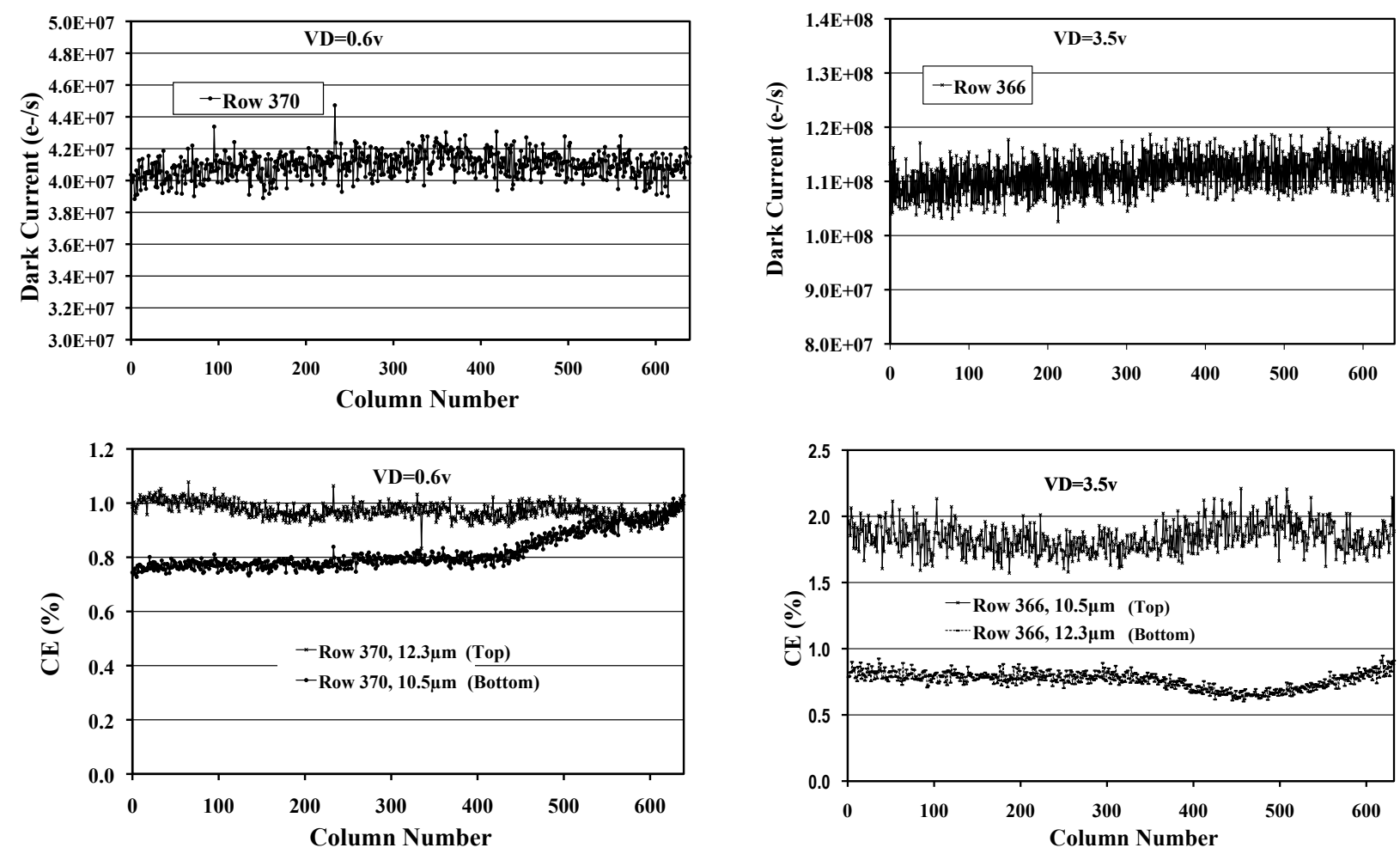

Figure 10. Top left: dark current for each pixel in row 370 (grating QWIP). Top right: dark current for row 366 (C- QWIP). Bottom: CE plots for corresponding arrays. Both arrays are the same as in the previous figures. $\mathrm{T}=43 \mathrm{~K}$ in all cases.

\subsection{Optical crosstalk}

An optical crosstalk experiment was performed on the C-QWIP which had a residual $\sim 45 \mu \mathrm{m}$ layer of GaAs substrate that had not been removed in the thinning process (all the QmagiQ arrays have the substrate completely removed). The concern was that this residual layer of GaAs material might provide a path where signal could be "light-piped" between neighboring pixels thereby reducing the image quality. A pinhole mask was designed and fabricated on a silicon wafer coated with aluminum/silicon dioxide and then patterned with a multitude of $15 \mu \mathrm{m}$ diameter holes separated by an increasing $4 \mu \mathrm{m}$ step pattern $(501 \mu \mathrm{m}$ between the first two pinholes then $505 \mu \mathrm{m}$ between the second and third pinholes and so on for the $18 \times 18$ grid). This die was then placed faced down in direct contact with the QWIP array surface covering about $80 \%$ of the QWIP array surface. The array was then illuminated with a hot plate set at $353 \mathrm{~K}$. The resulting image is shown below in figure 11. Assuming an array response out to $12.5 \mu \mathrm{m}$ it is expected that the diffraction would cause the pinhole to expand from $15 \mu \mathrm{m}$ to $29.6 \mu \mathrm{m}$ thus spilling into adjacent pixels. This diffraction limit is illustrated in the bottom frame of figure 11. The numerical values in each pixel correspond to the number of counts after background subtraction. It is clear that there is illumination in adjacent pixels but the signal is essentially 
the background signal in pixels outside the $3 \times 3$ immediate neighborhood. The outside pixels are not zero because the background fluctuation persists when a constant value is subtracted from every pixel.

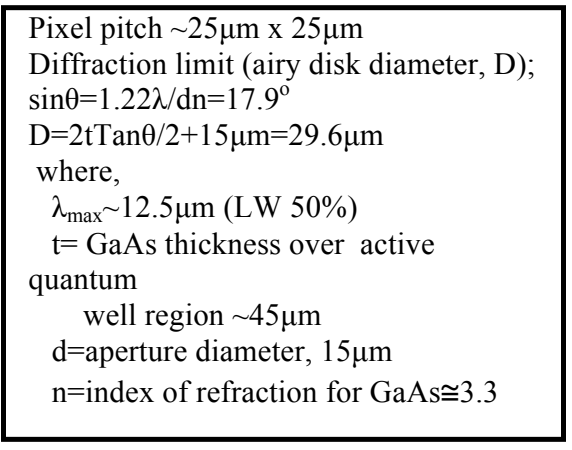

\subsection{Technology Readiness Level (TRL)}

An important and essential process for qualifying new or previously unused technology in a NASA space mission is the technology readiness level demonstration. There are nine levels with level 6 (TRL 6) being the level at which new hardware must be demonstrated. Typically, this means qualification in the environment which the instrument will be subjected through out the mission; radiation effects, vibration, thermal cycling and (in some cases) shock. Both the readout and QWIP hybrids were subjected to gamma, proton and heavy ion radiation equivalent to $35 \mathrm{krads}$ or almost 10 times the expected mission dose. At these levels and at the operating temperature of $43 \mathrm{~K}$ minimal effects were observed and none were considered to be a mission risk. A fully functioning focal plane assembly (shown in figure 5) was subjected to 40 thermal cycles from $300 \mathrm{~K}$ to $77 \mathrm{~K}$ and back to $300 \mathrm{~K}$. Every tenth cycle went to $43 \mathrm{~K}$ to collect the array performance data. After the completion of the 40 cycles there was essentially no change in any of the three QWIP arrays (2 grating QWIP hybrids and one C-QWIP hybrid). The final environmental test performed was vibration to simulate the effect of the launch. Since this is a qualification test the vibration loads are specified $3 \mathrm{db}$ above the expected loads. The focal plane assembly was subjected to a series of vibration input loads including $\mathrm{x}, \mathrm{y}$ and $\mathrm{z}$-axis random vibration for 2 minutes/axis, a sine sweep and sine burst test $(15 \mathrm{Gs}$ at $20 \mathrm{~Hz})$. No failures occurred and this assembly and the overall design were certified by an independent review panel as having met the requirements for TRL $6[10]$.

\section{Acknowledgements}

The authors would like to acknowledge the following individuals for their invaluable support to this NASA Landsat/TIRS project: Brent Mott, Tom Hartman, Larry Hess, Audrey Ewin, Ron Hu, Nick Costen, Fred Wang, Steve Snodgrass, Allen Lunsford, Nick Boehm, Roger Foltz, Emily Kan, Mike Hickey, Ed Wassel, Duncan Kahle, Bob Rosenberry, Greg Delo, Betsy Pugel, Sam Moseley, Avery Miles, Carol Sappington, Laddawan Miko, Trang Nguyen, Trinh Degumbia, Tomoko Adachi, Peter Shu, Jay Cho, Bing Guan, Phil Goodwin, Sherry Warner of NASA's Goddard Space Flight Center; Jason Sun of the Army Research Laboratory; Axel Reisinger, Rich Dennis, Kelly Patnaude, Doug 
Burrows, of QmagiQ, LLC and we would also like to express our appreciation to Jim Woolaway and Susan Petronio of Indigo Corp., Paul Pinsukanjana of Intelliepi, Corp. and Dave Hartzell of IQE Corp. for their ongoing support.

\section{References}

[1] See http://landsathandbook.gsfc.nasa.gov/handbook/handbook_htmls/chapter1/chapter1.html

[2] See http://landsat.gsfc.nasa.gov/news/news-archive/soc_0023.html

[3] Jhabvala, M., et al, " Development and operation of the microshutter array system", SPIE, Orlando, FL, March, (2008).

[4] Jhabvala, M., Choi, K., and La, A., " Development of a 1K x 1K, 8-12 micrometer QWIP Array ", Infrared Physics and Technology v42, Dana Point, CA, June, (2006).

[5] Jhabvala, M., Forrest, K. and Kaipa, R. "Gallium Arsenide Quantum Well Far IR Array Imager", Technology 2001, San Jose, CA, Dec 3-5, (1991).

[6] Jhabvala, M., Choi, K., Goldberg, A., La, A. and Gunapala, S. "Development of a 1K x1K GaAs QWIP Far IR Imaging Array", SPIE, San Diego, CA, August, (2003).

[7] User's Guide ISC9803 Standard 640 Advanced Readout Multiplexer, version 1.2.

[8] Choi, K., Lin, C., Leung, K., Tamir, T., Mao, J., Tsui, D. and M. Jhabvala, "Broadband and narrow band light coupling for QWIPs", ", Infrared Physics and Technology v44, Turin, Italy, October, (2002).

[9] Sundaram, M., and Wang, S., "Two-color QWIP FPAs" SPIE, Orlando, FL , April, (2000).

[10] Jhabvala, M. "Focal Plane Assembly Technology Readiness Level 6 Qualification Summary", NASA TIRS Report

TIRS-FPA-REF-0033, October, (2009). 